\title{
Investigation of Pionic Contribution in the Lepton and Antilepton Production Cross Section in p-Cu and p-Pt Collision
}

\author{
R. Rostami and F. Zolfagharpour \\ Department of Physics, University of Mohaghegh Ardabili, Ardabil, Iran \\ Correspondence should be addressed to F. Zolfagharpour; zolfagharpour@uma.ac.ir
}

Received 21 November 2015; Revised 28 March 2016; Accepted 3 April 2016

Academic Editor: Enrico Lunghi

Copyright (C) 2016 R. Rostami and F. Zolfagharpour. This is an open access article distributed under the Creative Commons Attribution License, which permits unrestricted use, distribution, and reproduction in any medium, provided the original work is properly cited. The publication of this article was funded by SCOAP ${ }^{3}$.

\begin{abstract}
For detailed explanation of the experimental results of lepton production cross section in hadronic collisions such as nucleonnucleon or nucleon-nuclei, it is of great importance to use quarks and sea quarks distribution function inside free and bound nucleons. In this paper, the role of pion cloud inside the nucleus in the structure function of $\mathrm{Cu}$ and Pt nuclei and the EMC ratio of these nuclei were investigated by using harmonic oscillator model. Then, in the framework of the Drell-Yan process and conventional nuclear theory, GRV's quarks distribution functions and pionic quarks distribution functions were used to calculate lepton and antilepton production cross section in $\mathrm{p}-\mathrm{Cu}$ and $\mathrm{p}-\mathrm{Pt}$ scattering. From the results and based on the mentioned model, by considering pionic contribution, the theoretical results are improved.
\end{abstract}

\section{Introduction}

Nuclei are made up of protons and neutrons bounded by strong nuclear forces, with the binding energy much less than the rest mass of the nucleons. Therefore, it is expected that scattering cross section of the leptons from nucleus be obtained from noncoherent summation of scattering cross sections from individual bound nucleons [1-5]. Hence this impulse approximation seems to explain the experimental data obtained from relativistic lepton-nucleus or hadronic collision. However, in 1983, the experiment done by a group of European researchers confronted scientists with experimental results which demonstrated that the structure of free and bound nucleons was different [6-8]. It was therefore concluded that some phenomena existed which were responsible for the slight difference in the internal structure of nucleons and consequently in quarks distribution functions in the bound nucleons compared to free nucleons. This change in quark distribution functions, which alters the free nucleons structure functions, is known as the EMC effect. The researches done by many other groups, such as Thomas and his colleagues in the following years $[1,9,10]$, showed that pure Fermi motion cannot explain the EMC effect and that binding energy plays an important role. Their effect is the result of nuclear interactions which play an important role in the deep inelastic scattering of leptons from nuclei. However it should be mentioned that the consideration of these phenomena alone is insufficient to explain the difference between quarks distribution functions inside bound and free nucleon. Ericson and Thomas [9] investigated the role of the pion field in the enhancement of the EMC effect in iron. Malace et al. [10] performed an overview of the EMC effect. In this paper, by using the works of mentioned authors and others $[3,9-13]$ to improve on previous results, the effect of Fermi motion and binding energy and pionic contribution was considered in extracting the quarks distribution functions inside the bound nucleon in the $\mathrm{Cu}$ and $\mathrm{Pt}$ nucleus, while other phenomena like shadowing effect and quark exchange were disregarded [9]. Therefore, the dileptons production cross section in $p+$ nucleus $\rightarrow l^{+}+l^{-}+x$ scattering can be calculated by using the mentioned effects in the framework of conventional nuclear theory and Drell-Yan process. The used model is the harmonic oscillator model that is modified by considering different $\hbar \omega$ parameters for occupied nucleon energy state inside nucleus. This modification is done since nucleons in deferent state may be affected by different main 
field. In the case of less binding energy, better agreement between the theoretical results and experimental data can be achieved compared with the case when the nucleons are affected by fixed main field [14-17]. GRV's quarks distribution functions for bound nucleons and pions are used for the calculation of quarks distribution inside nucleus [11]. In this work, to investigate the pionic contribution in the lepton and antilepton production cross section in $\mathrm{p}-\mathrm{Cu}$ and $\mathrm{p}-\mathrm{Pt}$ collision, by first considering the pionic contribution, the structure function of these nuclei and their corresponding EMC ratio were studied. Then their pionic contribution in quarks distribution functions was probed and finally the amount of this contribution in the dilepton production cross section in $\mathrm{p}-\mathrm{Cu}$ and $\mathrm{p}-\mathrm{Pt}$ scattering was computed.

\section{Calculation of the Structure Function and EMC Ratio of the ${ }^{63} \mathrm{Cu}$ and ${ }^{195} \mathrm{Pt}$ Nuclei}

The nucleus structure function by considering the pion cloud effect is defined as follows $[12,17]$ :

$$
\begin{aligned}
F_{2}^{A}\left(x, Q^{2}\right)= & \int_{x}^{A} f_{\pi}^{A}(z) F_{2}^{\pi}\left(\frac{x}{z}, Q^{2}\right) d z \\
& +\sum_{N=n, p} \sum_{n l} \int_{x}^{A} g_{n l}^{N} f_{N}^{A}(z)_{n l} F_{2}^{N}\left(\frac{x}{z}\right) d z,
\end{aligned}
$$

where the first term indicates the pionic contribution and the next term indicates the nucleon contribution in the nucleus structure function. $z=p_{n l} q / m_{N} q_{0}$ is the fraction of the total momentum of nucleus carried by nucleon in which $p_{n l}$ is the nucleon momentum in the state $n, l . q$ is the momentum transferred from the lepton to the nucleon and $q_{0}$ is the energy transferred from the lepton to the nucleon. $x=Q^{2} / 2 m_{N} q_{0}$ is the Bjorken scaling variable, where $Q^{2}$ is the square of the four-momentum transfer from the lepton. $F_{2}^{\pi}\left(x / z, Q^{2}\right)$ is the pion structure function and $F_{2}^{N}(x / z)$ is the structure function of free nucleons in which $F_{2}^{N=n}(x / z)$ and $F_{2}^{N=p}(x / z)$ refer to neutron and proton, respectively. $f_{\pi}^{A}(z)$ is the distribution function of pion inside the nucleus that is given as follows [17]:

$$
\begin{aligned}
& f_{\pi}^{A}(z)=\frac{3 g^{2}}{16 \pi^{2}} \Delta \lambda z\left[\frac{1}{\lambda} \exp \left[-2 \lambda \frac{t_{0}+m_{\pi}^{2}}{m_{\pi}^{2}}\right]\right. \\
& \left.+\frac{1}{2} E_{i}\left[-2 \lambda \frac{t_{0}+m_{\pi}^{2}}{m_{\pi}^{2}}\right]\right],
\end{aligned}
$$

where $E_{i}(-z)=-\int_{z}^{\infty}\left(e^{-t} / t\right) d t, t_{0}=\left|m_{N}^{2}\left(z^{2} /(1-z)\right)\right|, m_{\pi}=$ $139.570 \mathrm{MeV}$ is the pion mass, and $g=13.5$ is the coupling constant. The cut-off parameter $\lambda$ plays the most substantial role that shows changes in nuclear environments. When the nucleon is embedded in a nucleus, several modifications, such as the polarization of nuclear medium, occur. These may be expressed by an effective change of $\lambda$. By considering pions and ignoring the contribution made by virtual particles like $\Delta$ particle and heavy mesons, the momentum sum rule can be written as follows:

$$
\langle z\rangle_{N}+\eta_{\pi}=1
$$

where $\langle z\rangle_{N}$ and $\eta_{\pi}$ are the momentum carried by the nucleons and pions, respectively, and are defined as follows:

$$
\begin{aligned}
\langle z\rangle_{N} & =\frac{1}{A} \int_{x}^{A} z f_{N}^{A}(z)_{n l} d z, \\
\eta_{\pi} & =\int_{0}^{M_{A} / m_{N}} z f_{\pi}^{A}(z) d z,
\end{aligned}
$$

where $M_{A}$ and $m_{N}$ represent the mass of nucleus and the mass of nucleons, respectively. In the second term of (1), the first sum is related to the total number of neutrons and protons and the second is related to the quantum number of each energy state. $g_{n l}^{N}$ is the occupation number of energy state $\varepsilon_{n l}$ such that, for protons, $N=p$ and, for neutrons, $N=n$. Function $f_{N}^{A}(z)_{n l}$, which is calculated by the wave functions of harmonic oscillator, describes energy and momentum distribution of nucleons inside the nucleus as follows [12, 13]:

$$
\begin{aligned}
& f_{N}^{A}(z)_{n l}=\frac{1}{2}\left(\frac{m_{N}}{\hbar \omega}\right)^{1 / 2} \frac{n !}{\Gamma(n+l+3 / 2)} \\
& \quad \cdot \sum_{t_{1}=0}^{n} \sum_{t_{2}=0}^{n} \frac{(-1)^{t_{1}+t_{2}}}{t_{1}+t_{2}}\left(\begin{array}{c}
n+l+\frac{1}{2} \\
n-t_{1}
\end{array}\right)\left(\begin{array}{c}
n+l+\frac{1}{2} \\
n-t_{2}
\end{array}\right) \Gamma[l \\
& \left.\quad+t_{1}+t_{2}+1, \frac{m_{N}}{\hbar \omega}\left(z-1-\frac{\varepsilon_{n l}}{m_{N}}\right)^{2}\right],
\end{aligned}
$$

where $\varepsilon_{n l}$ is the mean one nucleon separation energy or, from the other point of view, is the average removal energy that has been considered the same for nucleons in different levels [13]. In this study, $\varepsilon_{n l}$ was considered to be different for nucleons in different levels.

$f_{N}^{A}(z)_{n l}$ should satisfy the normalization rule:

$$
\sum_{N=n} \sum_{n l} \int_{0}^{\infty} g_{n l}^{N} f_{N}^{A}(z)_{n l} d z=A .
$$

Taking into consideration the sea quarks and gluons contributions, the structure function of nucleons satisfies the sum rules:

$$
\int_{0}^{1} F_{2}^{N}(x) d x=1 .
$$

In the harmonic oscillator, $\hbar \omega$ in the neutral unit could be expressed as follows:

$$
\hbar \omega(\mathrm{MeV})=\frac{42.2}{\left\langle r^{2}\right\rangle_{n l}}\left(2 n+l+\frac{3}{2}\right),
$$

where $\left\langle r^{2}\right\rangle_{n l}$ is the mean square radius of state $n, l$ and its unit is Fermi. The EMC ratio as the ratio of the structure function of nucleus to the deuterium structure function is defined as follows:

$$
R_{\mathrm{EMC}}(x)=\frac{2 F_{2}^{A}\left(x, Q^{2}\right)}{A F_{2}^{2_{H}}\left(x, Q^{2}\right)} .
$$




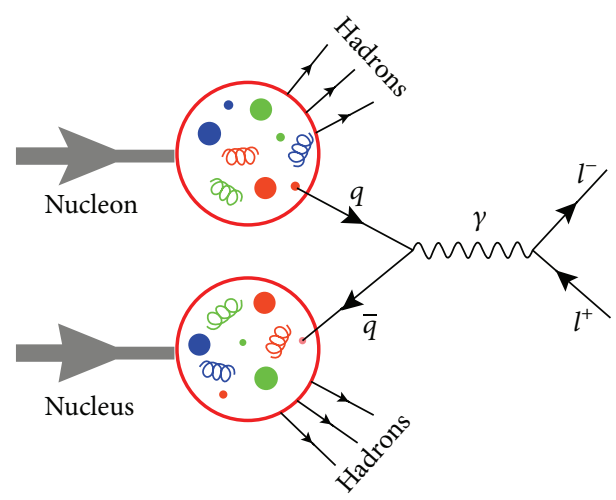

FIGURE 1: Drell-Yan process, a quark with fraction of momentum $x_{1}$ in the hadron $A$ and an antiquark with fraction of momentum $x_{2}$ in the hadron $B$ collide and annihilate to create a photon. This virtual photon with mass $m=\sqrt{x_{1} x_{2} s}$ decays into a lepton pair [18].

\section{Drell-Yan Process}

In 1970, initial studies of lepton pair production $\mu^{+} \mu^{-}$in Hadron-Hadron collision was reported by Christenson et al. [21] as follows:

$$
h_{A}+h_{B} \longrightarrow l^{+} l^{-}+x .
$$

The process of which is shown in Figure 1. The dilepton production cross section is decreased by increasing the dilepton mass. Drell and Yan [22] suggested that, in HadronHadron collision, leptons with opposite sign are produced. In the first stage, a quark of a hadron is annihilated with an antiquark of other hadrons and a virtual photon is generated. The generated photon is converted into lepton pairs with opposite sign like $\mu^{+} \mu^{-}$and $e^{+} e^{-}$. This is an electromagnetic process which can be calculated. Many research groups such as Jung and Miller have investigated deep inelastic scattering for different purposes in the framework of the Drell-Yan process [23]. The role of different phenomena, including Fermi motion and binding energy, together with the pionic contribution in nuclear Drell-Yan process has been probed by various case studies [24-26]. In this paper, in order to improve on previous results, the pionic contribution in the dilepton production cross section in the framework of the Drell-Yan process and conventional nuclear theory [3, 913] was calculated by using harmonic oscillator model. In order to predict the differential cross section of lepton pairs production in an interaction like Figure 1, an idea of the quarks and antiquarks distribution functions inside $A$ and $B$ hadrons was necessary. The lepton production cross section in the collision of two hadrons in terms of quarks and antiquarks distribution functions is calculated as follows [27]:

$$
\begin{aligned}
& \left(s \frac{d^{2} \sigma}{d \sqrt{\tau} d y}\right) \\
& \quad=\frac{8 \pi \alpha^{2}}{9 \tau^{3 / 2}} K \sum_{i} e_{i}^{2}\left[q_{i}^{A}\left(x_{1}\right) \bar{q}_{i}^{B}\left(x_{2}\right)+\bar{q}_{i}^{A}\left(x_{1}\right) q_{i}^{B}\left(x_{2}\right)\right],
\end{aligned}
$$

where $\sqrt{\tau}=m / \sqrt{s}=\sqrt{x_{1} x_{2}}, \sqrt{s}$ is the Hadron-Hadron c.m. energy, and $m=\sqrt{x_{1} x_{2} s}$ is the mass of dileptons.
$K$ is the correction coefficient known as $K$ factor that is considered in order to provide an agreement between the experimental and the theoretical results. $e_{i}$ is the fractional electric charge of quark $i . \bar{q}_{i}^{A}(x)$ is the $i$ th antiquark distribution function inside bound hadron $A$ and $q_{i}^{B}(x)$ is $i$ th quark distribution function inside the bound hadron $B$, which is defined as follows [28]:

$$
\begin{aligned}
q_{i}^{B}(x)= & \sum_{n l} \int_{x}^{A} \frac{1}{z} g_{n l}^{N} f_{N}^{A}(z)_{n l} q_{i}\left(\frac{x}{z}\right) d z \\
& +\int_{x}^{A} \frac{1}{z} f_{N}^{\pi}(z)_{n l} q_{i}^{\pi}\left(\frac{x}{z}\right) d z,
\end{aligned}
$$

where $q_{i}(x)$ and $q_{i}^{\pi}(x)$ are the distribution functions of quarks inside the free hadrons [11] and free pions [29].

\section{Results and Discussion}

According to (1), to calculate the share of nucleons and pions in the structure function of nucleus, it is required to calculate the distribution function of nucleons and pions inside the nucleus in addition to the free nucleon and pion structure function. In this work, to calculate the structure function of $\mathrm{Cu}$ and Pt nuclei, first the GRV's free neutron and free proton structure functions were applied and, using the parameters of Table 1 and according to (6), the nucleons distribution function was calculated. Then, in order to apply pionic contribution in the structure function of nucleus in addition to the share of nucleons, using the GRV's pion structure function and according to (2), the pion distribution function inside the nucleus was calculated.

Figure 2 shows the GRV's free neutron and proton structure function, which were applied in the calculations. The plotted graph for free proton structure function is in agreement with the experimental data that are taken from [19]. In this study, $\lambda=0.026$ and $m_{\pi}=139.570 \mathrm{MeV}$ was used [17]. From (3), $\langle z\rangle_{N}$ was calculated as 0.9675 and 0.9707 for $\mathrm{Cu}$ and Pt nuclei, respectively. Also From (4), $\eta_{\pi}$ was calculated as 0.0325 and 0.0299 for $\mathrm{Cu}$ and Pt nuclei, respectively. Convenient $\Delta \lambda$ for ${ }^{2} \mathrm{H},{ }^{63} \mathrm{Cu}$, and ${ }^{195} \mathrm{Pt}$ nuclei was considered $0.00242,0.00792$, and 0.00861 , respectively, to be satisfactory for (5). Figure 3 shows a plot of the calculated distribution function of pion inside ${ }^{2} \mathrm{H},{ }^{63} \mathrm{Cu}$, and ${ }^{195} \mathrm{Pt}$ nuclei. As shown in the figure, for all nuclei in about $z=0.35$, the distribution function of pions has a peak and its amount is greater for heavier nucleus. The distribution function of pions, which carry a very low or high fraction of the total momentum of nucleus, tends to zero. Figure 4 shows the GRV's LO and NLO pion structure function. The structure function of pion calculated according to the equation $F_{2}^{\pi}(x)$ [20] was plotted for comparison. The GRV's LO pion structure function was used in the calculations.

Figures 5 and 6 show plots of the structure function per nucleon of $\mathrm{Cu}$ and Pt nuclei in mean $Q^{2}=5 \mathrm{GeV}^{2}$ and the EMC ratio of these nuclei, by and without considering the pionic contribution. The dashed line shows the structure functions of $\mathrm{Cu}$ and $\mathrm{Pt}$ nuclei and their corresponding EMC ratio by considering the Fermi motion and the binding energy 


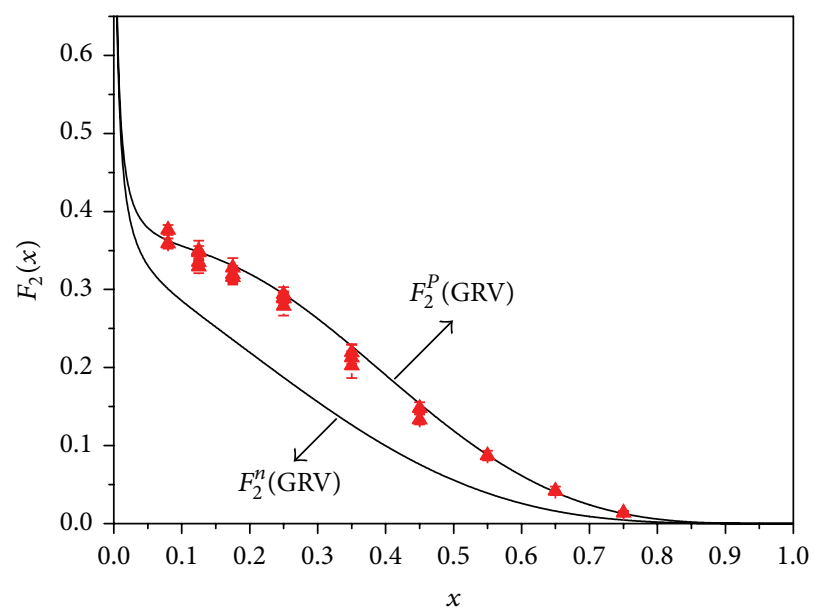

FIGURE 2: GRV's free neutron and proton structure function. The experimental data shown for free proton structure function are taken from [19].

TABLE 1: Brackets contain $\left\langle r^{2}\right\rangle^{1 / 2}$ (Fermi), $\hbar \omega(\mathrm{MeV}), g_{n l}^{p}, g_{n l}^{n}$, and $\varepsilon_{n l}$ $(\mathrm{MeV})$ parameters used in calculating the structure function of ${ }^{63} \mathrm{Cu}$ and ${ }^{195} \mathrm{Pt}$ nuclei for shells with quantum number $n, l$, respectively. $\left\langle r^{2}\right\rangle^{1 / 2}$ is taken from [30] for each level.

\begin{tabular}{|c|c|c|c|}
\hline \multirow{2}{*}{ Shell } & \multicolumn{3}{|c|}{ Nucleus } \\
\hline & ${ }^{2} \mathrm{H}$ & ${ }^{63} \mathrm{Cu}$ & ${ }^{195} \mathrm{Pt}$ \\
\hline $0 \mathrm{~s}$ & $\begin{array}{c}(2.09,15.35,1,1 \\
-1.5)\end{array}$ & $\begin{array}{c}(1.67,22.23,2,2, \\
-33)\end{array}$ & $(1.67,22.23,2,2,-34)$ \\
\hline $0 \mathrm{p}$ & & $\begin{array}{c}(2.44,17.34,6,6 \\
-32)\end{array}$ & $(2.44,17.34,6,6,-33)$ \\
\hline $0 \mathrm{~d}$ & & $\begin{array}{c}(3.10,12.51,10,10 \\
-31)\end{array}$ & $\begin{array}{c}(3.10,12.51,10,10 \\
-32)\end{array}$ \\
\hline 1s & & $\begin{array}{c}(3.48,11.95,2,2, \\
-30)\end{array}$ & $(3.48,11.95,2,2,-31)$ \\
\hline Of & & $\begin{array}{c}(3.95,11.92,9,14 \\
-29)\end{array}$ & $\begin{array}{c}(3.95,11.92,14,14 \\
-30)\end{array}$ \\
\hline $1 \mathrm{p}$ & & & $(4.44,11.39,6,6,-29)$ \\
\hline $0 g$ & & & $\begin{array}{c}(4.49,11.28,18,18 \\
-28)\end{array}$ \\
\hline $1 \mathrm{~d}$ & & & $\begin{array}{c}(4.55,10.98,10,10 \\
-27)\end{array}$ \\
\hline $2 \mathrm{~s}$ & & & $(6.67,10.64,2,2,-26)$ \\
\hline $\mathrm{Oh}$ & & & $\begin{array}{c}(5.15,10.32,8,22 \\
-25)\end{array}$ \\
\hline If & & & $\begin{array}{c}(5.23,10.02,0,14 \\
-24)\end{array}$ \\
\hline $2 \mathrm{p}$ & & & $(5.41,9.37,0,6,-23)$ \\
\hline $0 \mathrm{i}$ & & & $(5.43,9.30,0,5,-22)$ \\
\hline
\end{tabular}

according to $\varepsilon_{n l}$ which is listed in Table 1 . According to [12,1417], the EMC ratio of some nuclei was plotted by considering $\varepsilon_{n l}=-40 \mathrm{MeV}$ and different $\varepsilon_{n l}$ for nucleons of each level, respectively. The extracted results in [14-17] are compatible with the experimental data.

The results showed that, for $x=0.15$, pion cloud increased the structure function to about $7.9 \%$ for ${ }^{63} \mathrm{Cu}$ and about $8.8 \%$ for ${ }^{195} \mathrm{Pt}$, respectively. Also, for $x=0.15$ and by considering pionic contribution, the EMC ratio of ${ }^{63} \mathrm{Cu}$ and ${ }^{195} \mathrm{Pt}$ nuclei was increased to $5.44 \%$ and $6.28 \%$, respectively. According to [31], this contribution for deuteron and threebody nuclei was calculated to be less than $5 \%$. According to the extracted results from Figures 5 and 6, most contribution in the ${ }^{63} \mathrm{Cu}$ and ${ }^{195} \mathrm{Pt}$ nuclei structure function and their EMC ratio is related to Fermi motion. In small $x$, the role of pionic contribution is considerable in comparison with the role of binding energy. In mean $x$, the role of binding energy is considerable while the effect of pionic contribution is about zero and is not comparable with the binding energy effect. Finally, in large $x$, pionic and binding energy contribution in the ${ }^{63} \mathrm{Cu}$ and ${ }^{195} \mathrm{Pt}$ nuclei structure function and their EMC ratio is the same and about zero. To calculate pionic contribution in dilepton production cross section in hadronic collisions, it was necessary to first calculate quarks and sea quarks distribution functions inside nucleus by considering the pionic contribution. In Figures 7 and 8, by using GRV's quarks distribution functions in nucleons and pions, the quarks and sea quarks distribution functions inside ${ }^{63} \mathrm{Cu}$ and ${ }^{195} \mathrm{Pt}$ nuclei have been calculated.

Figure 9 shows dilepton production cross section in $\mathrm{p}$ $\mathrm{Cu}$ scattering in terms of $\sqrt{\tau}$ by and without considering the pionic contribution for $y=-0.2,0,0.1$, and 0.4 and $\sqrt{s}=38.8 \mathrm{GeV}$ and by considering $K$ factor. The full curve is obtained by considering the pionic contribution and the dashed line is plotted without considering the pionic contribution. In Figure 10, by and without considering the pionic contribution, the lepton and antilepton production cross section in p-Pt collision for $y=0.025,0.163$, and 0.6 and $\sqrt{s}=38.8 \mathrm{GeV}$ have been plotted by considering $K$ factor. The amount of used $K$ factor is indicated in Figures 9 and 10. Results show that $K$ factor increased linearly with increasing rapidity $y$. The linear fit of the $K$ factor in terms of $y$ for ${ }^{63} \mathrm{Cu}$ and ${ }^{195} \mathrm{Pt}$ nuclei is obtained as follows:

$$
\begin{aligned}
& K=\frac{3}{4} y+1.7 \quad \text { for } \mathrm{Cu}, \\
& K=0.3 y+1.87 \quad \text { for Pt. }
\end{aligned}
$$




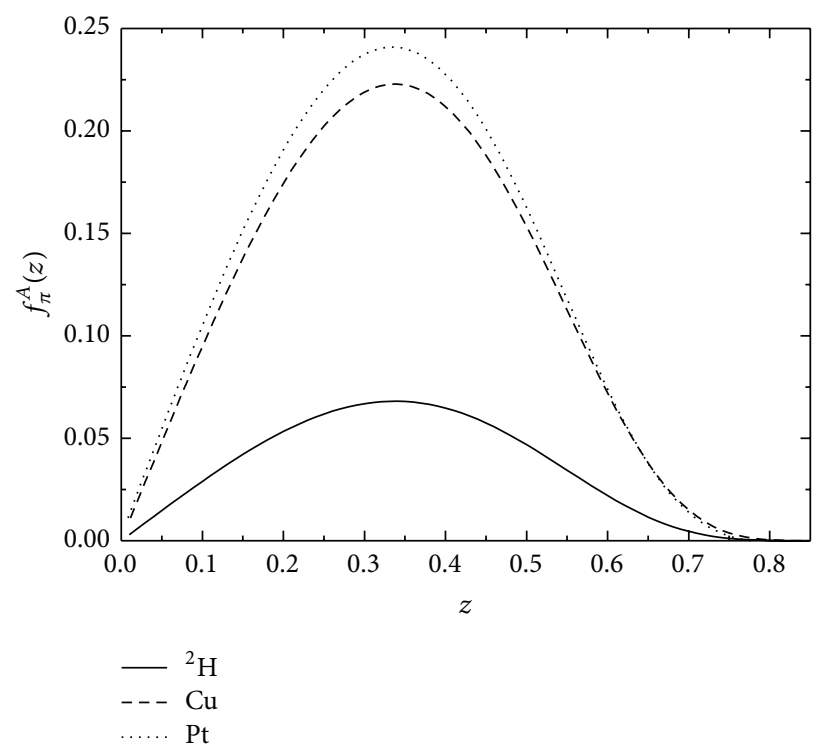

Figure 3: The distribution function of pion inside ${ }^{2} \mathrm{H},{ }^{63} \mathrm{Cu}$, and ${ }^{195} \mathrm{Pt}$ nuclei.

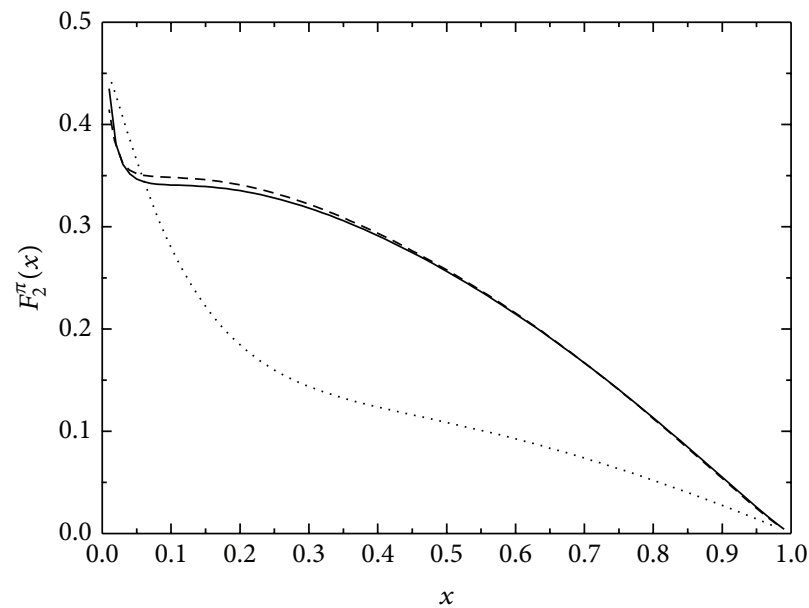

FIGURE 4: The structure function of pion. The full and dashed curve are the GRV's LO and NLO pion structure function. The dotted line is the structure function calculated according to equation of $F_{2}^{\pi}(x)$ in [20].

According to [27] different amount for $K$ factor has been reported by many research group. Pionic cloud resulted in an increase in the dileptons production cross section to about $3-8 \%$ in $\mathrm{p}-\mathrm{Cu}$ collision and about $3-6 \%$ in $\mathrm{p}-\mathrm{Pt}$ collision in available experimental data ranges.

As shown in Figures 9 and 10, in the production of dileptons, the effect of Fermi motion is considerable in comparison with the other phenomena and gradually decreases with increasing $\sqrt{\tau}$. Although the effect of pionic cloud in the production of dileptons is not significant, in small amounts of $\sqrt{\tau}$, its contribution (about $3-8 \%$ ) in comparison with the binding energy (about $0 \%$ ) is considerable. However, with increasing $\sqrt{\tau}$, the effect of pionic cloud in the production of dileptons decreases gradually and tends to zero.

\section{Conclusions}

In this study, by using the GRV's quarks distribution functions of nucleons and pions, lepton and antilepton production cross section in $\mathrm{p}-\mathrm{Cu}$ and $\mathrm{p}$ - $\mathrm{Pt}$ collision was investigated. For this purpose, the pionic contribution in the structure function of $\mathrm{Cu}$ and $\mathrm{Pt}$ nuclei and their corresponding EMC ratio was first calculated. The results showed that for $x=0.15$ the pionic contribution increased the structure function of ${ }^{63} \mathrm{Cu}$ and ${ }^{195} \mathrm{Pt}$ to $7.9 \%$ and $8.8 \%$, respectively. Also, for $x=$ 0.15 , and by considering pionic contribution, the EMC ratio of ${ }^{63} \mathrm{Cu}$ and ${ }^{195} \mathrm{Pt}$ nuclei was increased to $5.44 \%$ and $6.28 \%$, respectively. The results showed that pion cloud increased the dileptons production cross section up to about $3-8 \%$ in $\mathrm{p}-\mathrm{Cu}$ 


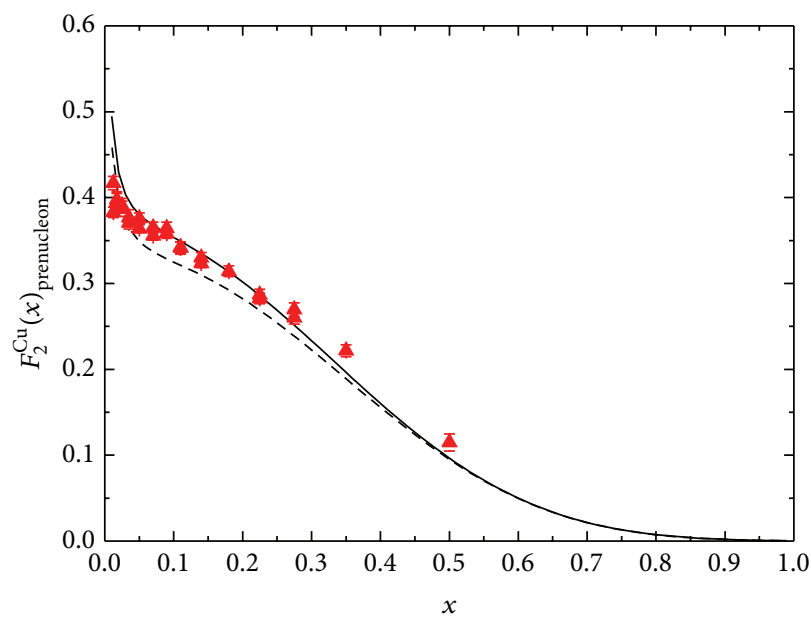

(a)

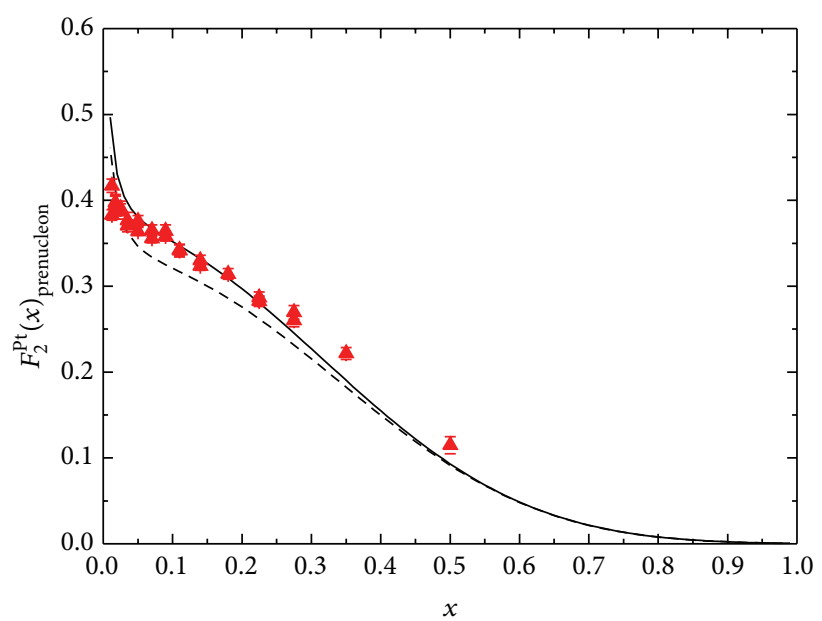

(b)

Figure 5: (a) and (b) show ${ }^{63} \mathrm{Cu}$ and ${ }^{195} \mathrm{Pt}$ structure functions per nucleon in mean $Q^{2}=5 \mathrm{GeV}^{2}$. The full curve is obtained by considering, the Fermi motion, the binding energy, and the pionic contribution effect. The dashed line shows the structure function by considering the Fermi motion and the binding energy. Experimental data shows deuterium structure function per nucleon [19].

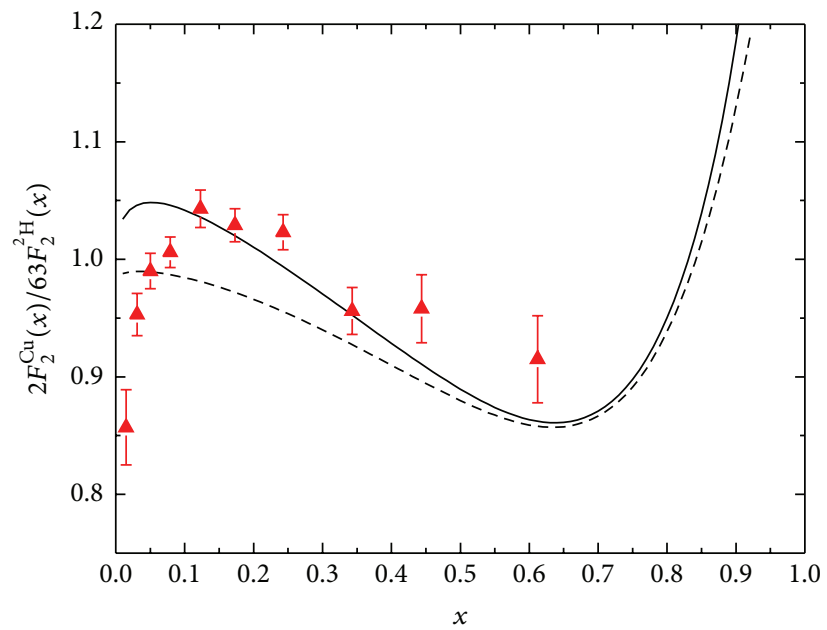

(a)

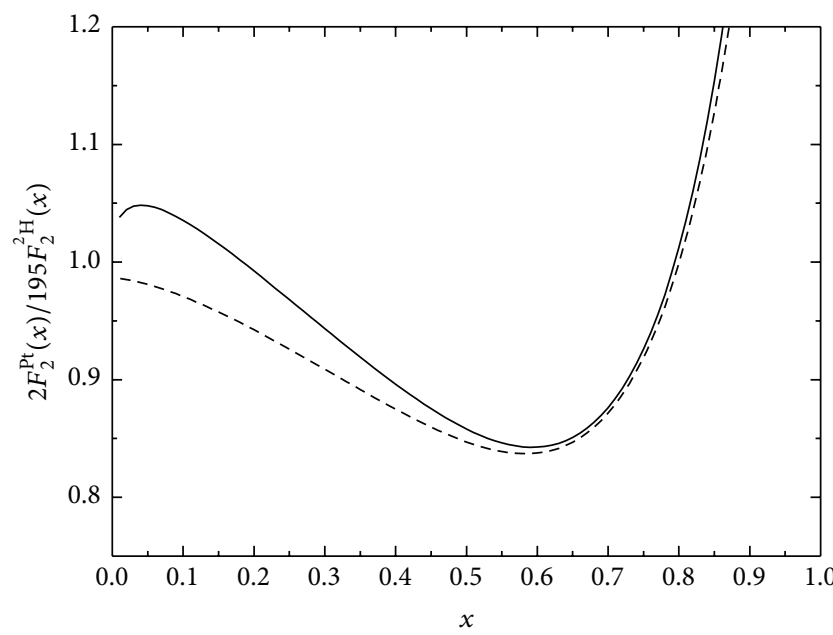

(b)

Figure 6: (a) and (b) show the ratio $R=2 F_{2}^{A} / A F_{2}^{2 H}$ in terms of $x$ for $\mathrm{Cu}$ and Pt nuclei. The full curve shows the results by considering the Fermi motion, the binding energy, and the pionic contribution effect. The dashed line shows the results by considering the Fermi motion and the binding energy. Experimental data are taken from [19].

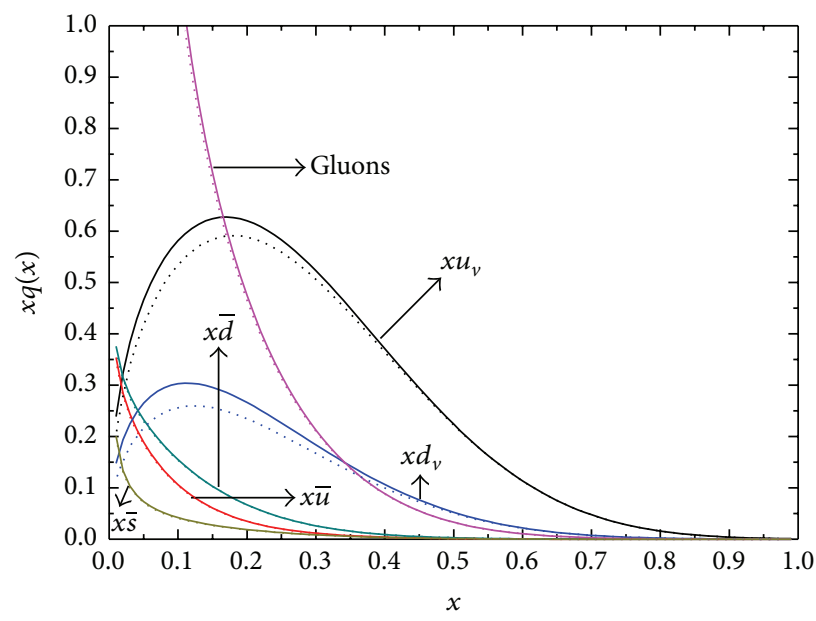

FIGURE 7: Distribution function of valance and sea quarks inside ${ }^{63} \mathrm{Cu}$ nucleus at $Q^{2}=20 \mathrm{GeV}^{2}$. The full curve is obtained by considering the pionic contribution and the dashed line is plotted without considering the pionic contribution. 


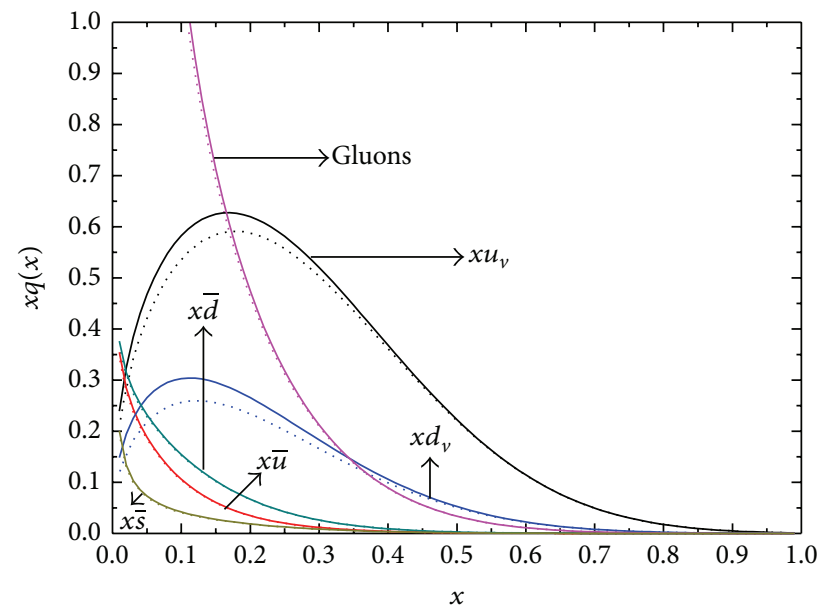

FIGURE 8: Distribution function of valance and sea quarks inside ${ }^{195} \mathrm{Pt}$ nucleus at $Q^{2}=20 \mathrm{GeV}^{2}$. The full curve is obtained by considering the pionic contribution and the dashed line is plotted without considering the pionic contribution.
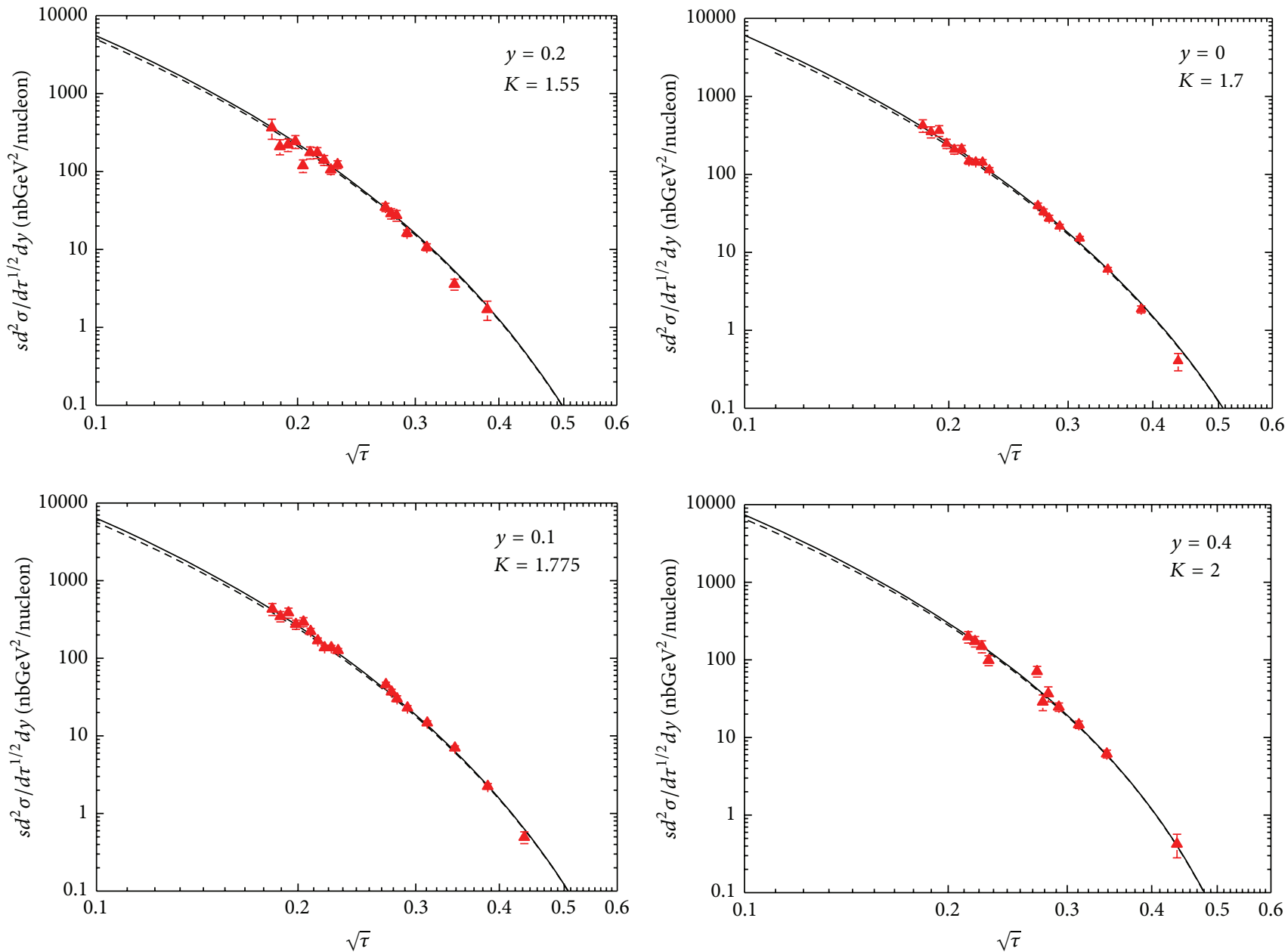

Figure 9: Dileptons production cross section for $\mathrm{p}-\mathrm{Cu}$ collision in terms of $\sqrt{\tau}$ according to (13) for $y=-0.2,0,0.1$, and 0.4 and $\sqrt{s}=38.8 \mathrm{GeV}$. The full curve is obtained by considering the pionic contribution and the dashed line is plotted without considering the pionic contribution. The used $K$ factor has been indicated in each graph. The difference between full and dashed lines is about 3-8\%. The experimental results have been taken from [19]. 

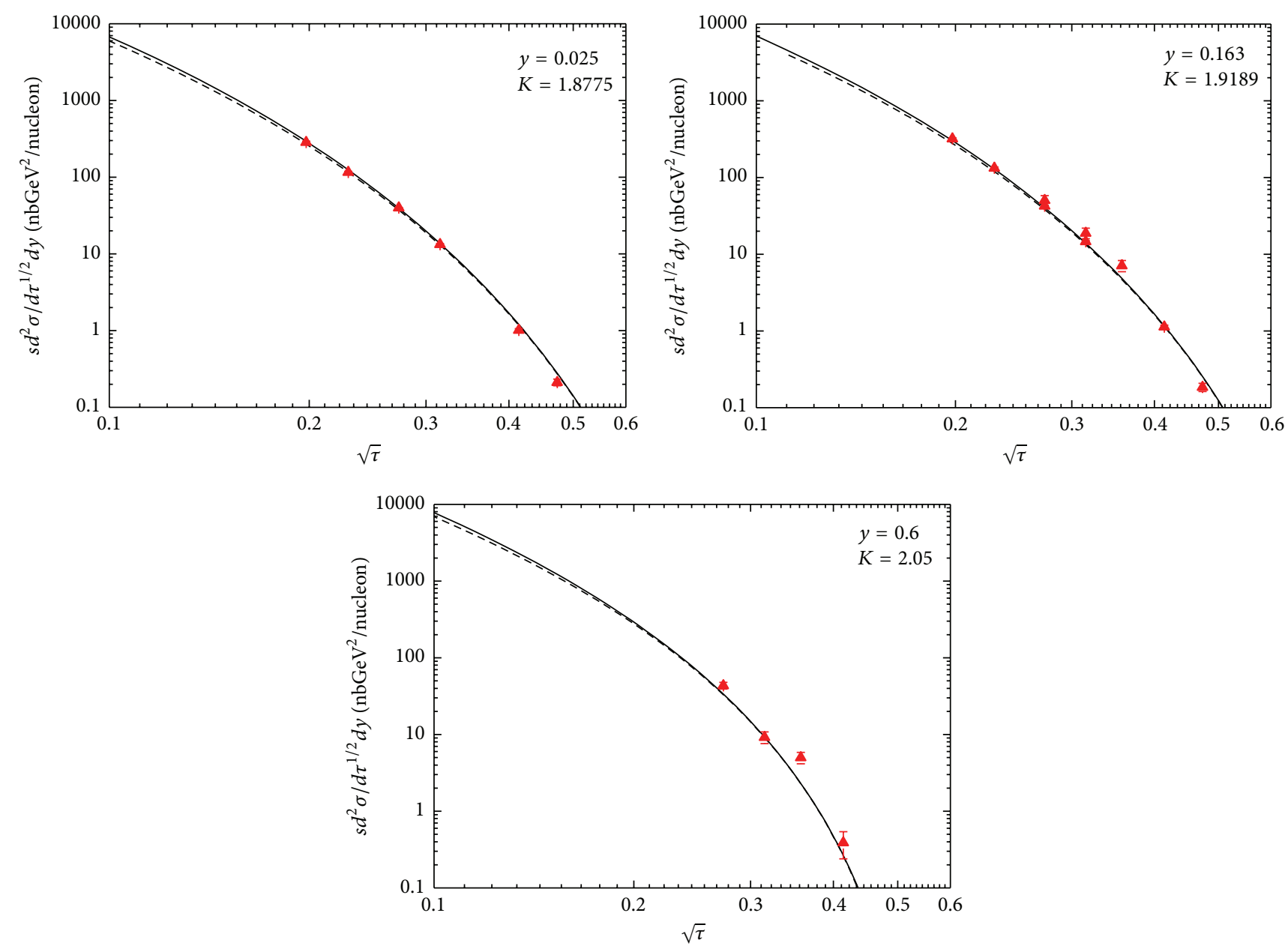

Figure 10: Dileptons production cross section for p-Pt collision in terms of $\sqrt{\tau}$ according to (13) for $y=0.025,0.163$, and 0.6 and $\sqrt{s}=38.8 \mathrm{GeV}$. The full curve is obtained by considering the pionic contribution and the dashed line is plotted without considering the pionic contribution. The used $K$ factor has been indicated in each graph. The difference between full and dashed lines is about 3-6\%. The experimental results have been taken from [19].

collision and about $3-6 \%$ in p-Pt collision. Comparison of the extracted results with the experimental data showed that these percentages for pionic contribution are appropriate.

\section{Competing Interests}

The authors declare that they have no competing interests.

\section{References}

[1] R. P. Bickerstaff and A. W. Thomas, "The EMC effect-with emphasis on conventional nuclear corrections," Journal of Physics G: Nuclear and Particle Physics, vol. 15, no. 10, pp. 15231569, 1989.

[2] G. Piller and W. Weise, "Nuclear deep-inelastic lepton scattering and coherence phenomena," Physics Report, vol. 330, no. 1, pp. 1-94, 2000.

[3] K. Saito and T. Uchiyama, "Effect of the Fermi motion on nuclear structure functions and the EMC effect," Zeitschrift für Physik A Atoms and Nuclei, vol. 322, no. 2, pp. 299-307, 1985.
[4] M. Arneodo, "Nuclear effects in structure functions," Physics Reports, vol. 240, no. 5-6, pp. 301-393, 1994.

[5] L. Jaffe, "Deep inelastic scattering with application to nuclear targets," in Proceedings of the Los Alamos Workshop, C85-06-10, 82 pages, 1985.

[6] J. J. Aubert, G. Bassompierre, K. H. Becks et al., "The ratio of the nucleon structure functions $\mathrm{F}_{2}^{N}$ for iron and deuterium," Physics Letters B, vol. 123, no. 3-4, pp. 275-278, 1983.

[7] A. Bodek, N. Giokaris, W. B. Atwood et al., "Electron scattering from nuclear targets and quark distributions in nuclei," Physical Review Letters, vol. 50, no. 19, pp. 1431-1434, 1983.

[8] A. Bodek, N. Giokaris, W. B. Atwood et al., "Comparison of the deep-inelastic structure functions of deuterium and aluminum nuclei," Physical Review Letters, vol. 51, no. 7, pp. 534-537, 1983.

[9] M. Ericson and A. W. Thomas, "Pionic corrections and the EMC enhancement of the sea in iron," Physics Letters B, vol. 128, no. 1-2, pp. 112-116, 1983.

[10] S. Malace, D. Gaskell, D. W. Higinbotham, and I. C. Cloët, "The challenge of the EMC effect: existing data and future directions," International Journal of Modern Physics E, vol. 23, no. 8, Article ID 1430013, 2014. 
[11] M. Glück, E. Reya, and A. Vogt, "Dynamical parton distributions of the proton and small-x physics," Zeitschrift für Physik C Particles and Fields, vol. 67, no. 3, pp. 433-447, 1995.

[12] S. V. Akulinichev, S. Shlomo, S. A. Kulagin, and G. M. Vagradov, "Lepton-nucleus deep-inelastic scattering," Physical Review Letters, vol. 55, no. 21, pp. 2239-2241, 1985.

[13] S. V. Akulinichev, S. A. Kulagin, and G. M. Vagradov, "The role of nuclear binding in deep inelastic lepton-nucleon scattering," Physics Letters B, vol. 158, no. 6, pp. 485-488, 1985.

[14] S. Hatampanah, N. S. Nikkhoo, and F. Zolfagharpour, "The role of pion cloud in the structure function and the EMC effect of ${ }^{27} \mathrm{Al},{ }^{56} \mathrm{Fe},{ }^{63} \mathrm{Cu}$ and, ${ }^{107} \mathrm{Ag}$ nuclei," American Journal of Modern Physics, vol. 2, no. 4, pp. 190-194, 2013.

[15] N. S. Nikkhoo and F. Zolfagharpour, "Study of the EMC effect for ${ }^{27} \mathrm{Al},{ }^{56} \mathrm{Fe},{ }^{63} \mathrm{Cu}$, and ${ }^{107} \mathrm{Ag}$ nuclei," Journal of Modern Physics, vol. 3, no. 11, pp. 1830-1834, 2012.

[16] M. Musavi, N. Nikkhoo, and F. Zolfagharpour, "Lepton scattering cross section and nuclear structure function of ${ }^{4} \mathrm{He}$ and ${ }^{12} \mathrm{C}$ nuclei," World Journal of Nuclear Science and Technology, vol. 3, pp. 131-135, 2013.

[17] N. Hamdolahi, F. Zolfagharpour, and N. Nikkhoo, "Differential cross section of electron scattering from ${ }^{3} \mathrm{He}$ and ${ }^{3} \mathrm{H}$ nuclei by considering pionic contribution," World Journal of Nuclear Science and Technology, vol. 2, no. 4, pp. 150-153, 2012.

[18] G. Moreno, C. N. Brown, W. E. Cooper et al., "Dimuon production in proton-copper collisions at $\sqrt{s}=38.8 \mathrm{GeV}$," Physical Review D, vol. 43, no. 9, pp. 2815-2835, 1991.

[19] http://durpdg.dur.ac.uk/.

[20] K. Nakano, "Remarks on the pionic constraints in the EMC effect," Journal of Physics G: Nuclear and Particle Physics, vol. 17, no. 11, pp. L201-L207, 1991.

[21] J. H. Christenson, G. S. Hicks, L. M. Lederman, P. J. Limon, B. G. Pope, and E. Zavattini, "Observation of massive muon pairs in hadron collisions," Physical Review Letters, vol. 25, no. 21, pp. 1523-1526, 1970.

[22] S. D. Drell and T.-M. Yan, "Massive lepton-pair production in hadron-hadron collisions at high energies," Physical Review Letters, vol. 25, no. 5, pp. 316-320, 1970.

[23] H. Jung and G. A. Miller, "Pionic contributions to deep inelastic nuclear structure functions," Physical Review $C$, vol. 41, no. 2, pp. 659-664, 1990.

[24] E. Marco and E. Oset, "Mesonic and binding contributions to the nuclear Drell-Yan process," Nuclear Physics A, vol. 645, no. 2, pp. 303-313, 1999.

[25] D. Dutta, J. C. Peng, I. C. Cloët, and D. Gaskell, "Pion-induced Drell-Yan processes and the flavor-dependent EMC effect," Physical Review C, vol. 83, no. 4, Article ID 042201, 2011.

[26] C. Chang and H. Li, "Glauber gluons in pion-induced Drell-Yan processes," Physics Letters B, vol. 726, no. 1-3, pp. 262-265, 2013.

[27] I. R. Kenyon, “The Drell-Yan process," Reports on Progress in Physics, vol. 45, no. 11, pp. 1261-1315, 1982.

[28] P. Amore, "Quark distribution functions in nuclear matter," Journal of Physics G: Nuclear and Particle Physics, vol. 27, no. 9, pp. 1905-1915, 2001.

[29] M. Glück, E. Reya, and I. Schienbein, "Pionic parton distributions revisited," European Physical Journal C, vol. 10, no. 2, pp. 313-317, 1999

[30] R. C. Barratt and D. F. Jackson, Nuclear Sizes and Structure, Oxford University Press, Oxford, UK, 1977.
[31] T. Uchiyama and K. Saito, "European Muon Collaboration effect in deuteron and in three-body nuclei," Physical Review C, vol. 38 , no. 5, pp. 2245-2250, 1988. 

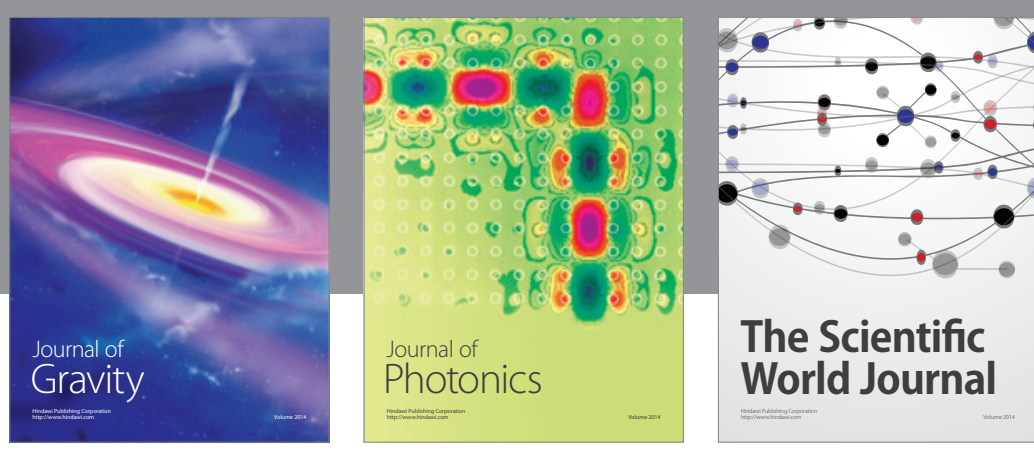

The Scientific World Journal
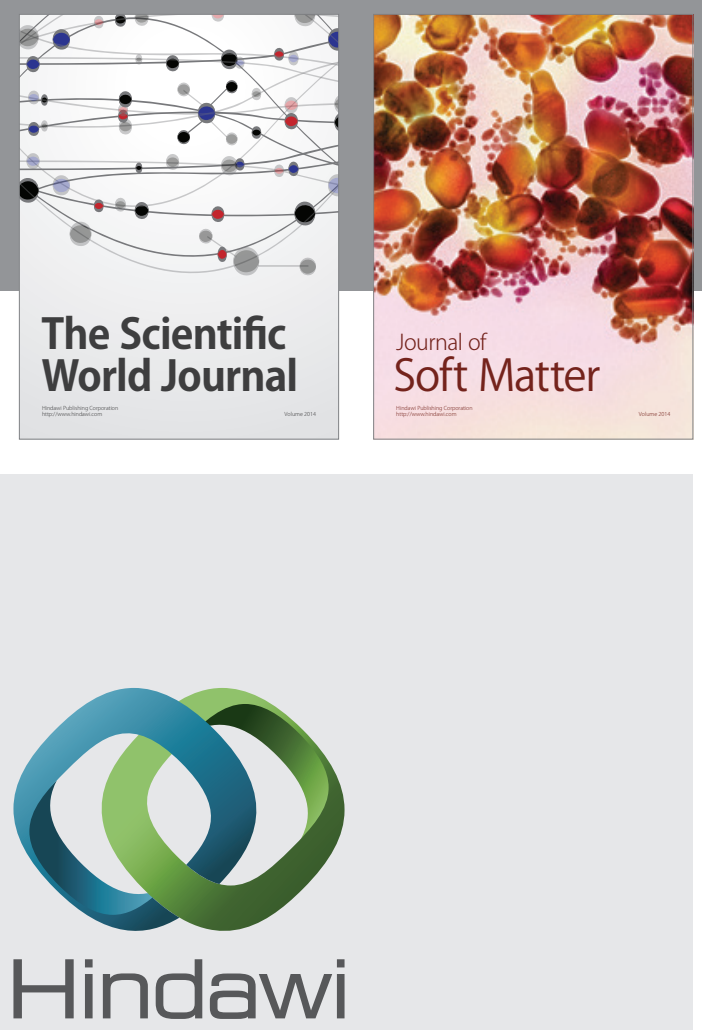

Submit your manuscripts at

http://www.hindawi.com

nternational Journal of

Statistical Mechanics
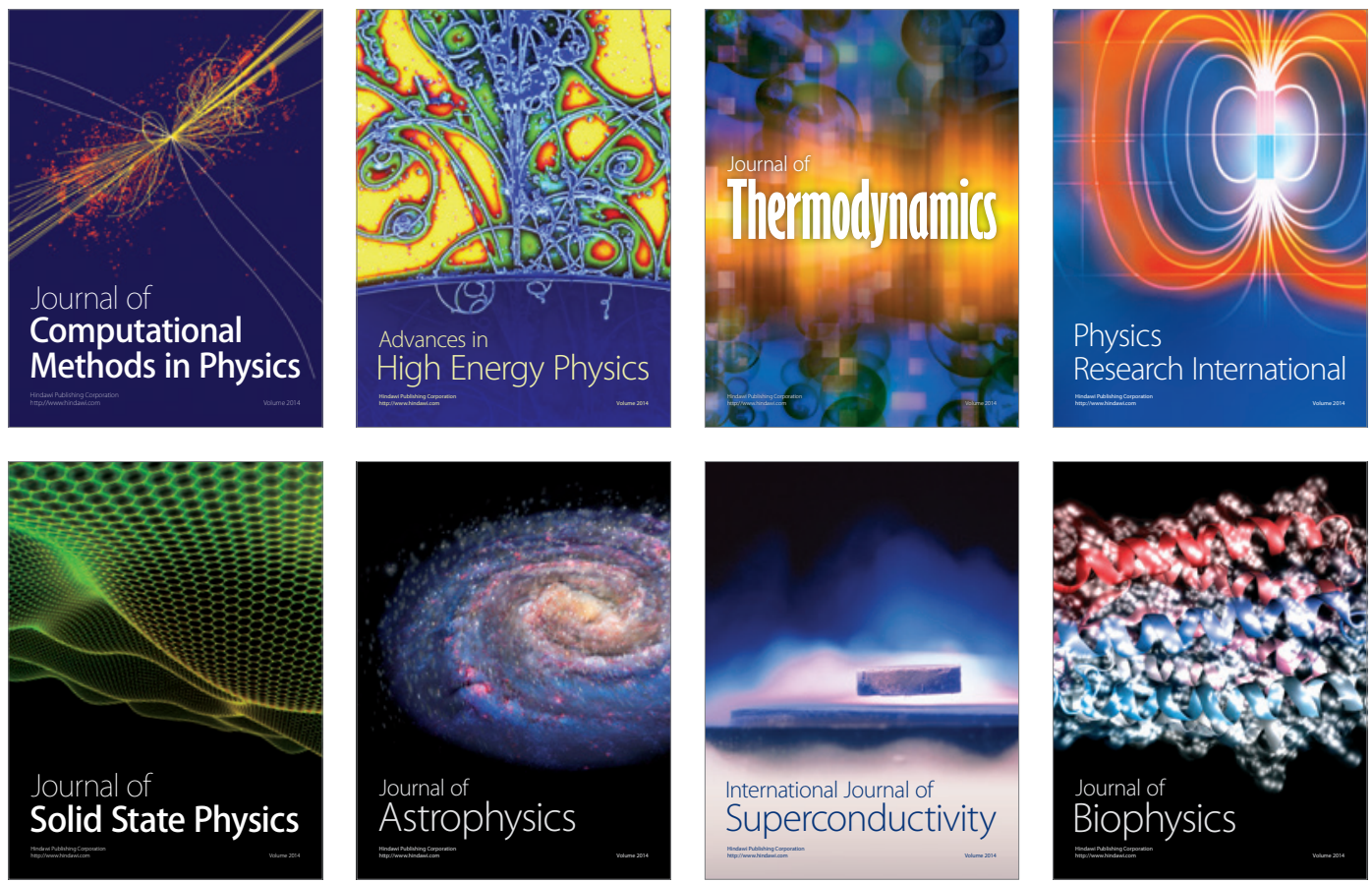
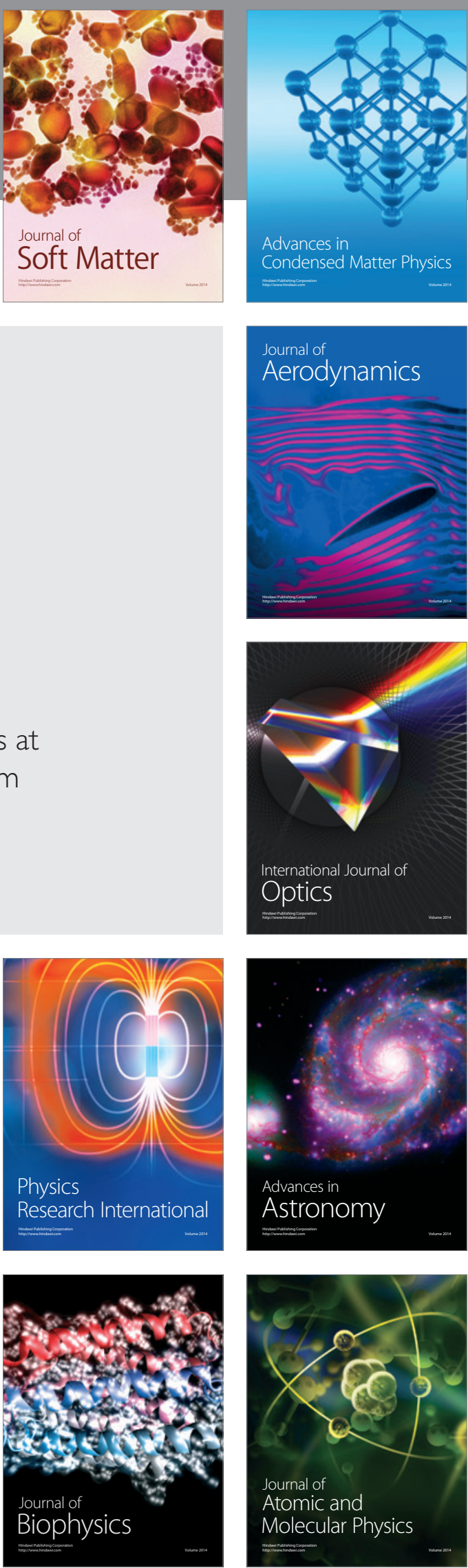